\title{
Estimation models for the Refractive Index response curve of EFBGs
}

\author{
Jean F. Kuhne, Ana M. Rocha, Rafael C. Barreto, and Ricardo C. Kamikawachi
}

\begin{abstract}
Estimation models for the surrounding refractive index response of EFBGs were developed from computer simulations and tested on experimental data. Numerical simulations were used to investigate the fundamental core mode's effective refractive index dependence with the fiber radius and the surrounding refractive index. An empirical mathematical model was developed upon the simulation results, and it was used to derive the estimation models. The response curve is estimated from a reference calibration curve using two different approaches: interpolation and extrapolation. The interpolation is performed using the wavelength response of the EFBGs at two different surrounding refractive index values. Then, the response curve
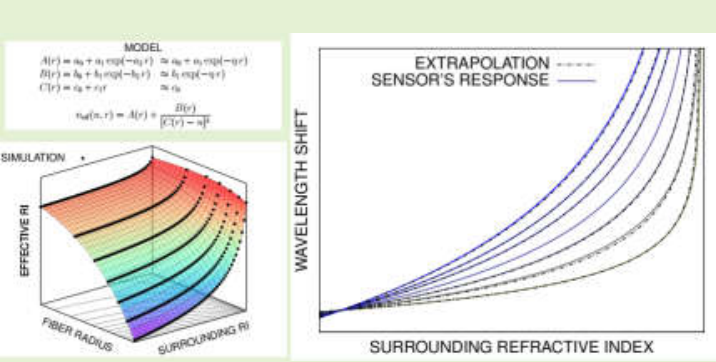

is obtained solving a simple pair of linear equations. The extrapolation is performed using the wavelength shift observed during the etching process, which is used to derive a multiplicative factor for the calibration curve. The response curves estimated by the model were compared with the experimental results, showing good agreement within the experimental uncertainties. Considering the surrounding refractive index ranging from $1.333 \mathrm{RIU}$ to $1.458 \mathrm{RIU}$, with the sensors' response ranging from $1544.84 \mathrm{~nm}$ to $1552.70 \mathrm{~nm}$, the maximum deviation between the estimation and the experimental results were $0.26 \mathrm{~nm}$ and $0.41 \mathrm{~nm}$, and the maximum standard deviation were $0.03 \mathrm{~nm}$ and $0.05 \mathrm{~nm}$, respectively for interpolation and extrapolation.
\end{abstract}

Index Terms-estimation, etched fiber Bragg grating, modelling, optical fiber sensor, refractive index measurement

\section{INTRODUCTION}

$\mathbf{E}$ TCHED fiber Bragg gratings (EFBG) have been applied as refractive index sensors since the first demonstration of their tunability through a controlled etching process [1]. In the early years of the 21 st century, the potential application of EFBGs as a refractive index sensor has attracted great interest from the optical fiber sensor community. Iadicicco et al.'s seminal work is a remarkable example, in which they presented experimental, numerical and theoretical results in the study of refractive index sensors based on FBGs inscribed in

Manuscript received month $x x, 2020$. This work was supported in part by the Coordenação de Aperfeiçoamento de Pessoal de Nível Superior (CAPES), Brazil, under Grant 001, in part by the Conselho Nacional de Desenvolvimento Científico e Tecnológico (CNPq) under Grant 443134/2014-6, in part by Financiadora de Estudos e Projetos (FINEP), in part by the Fundação Araucária, in part by Fundação para a Ciência e Tecnologia (FCT) and by the European Regional Development Fund (FEDER), through the Competitiveness and Internationalization Operational Programme (COMPETE 2020) of the Portugal 2020 framework under the projects MCTechs (POCl-01-0145-FEDER-029282), UIDB/50008/2020-UIDP/50008/2020. The associate editor coordinating the review of this article and approving it for publication was Dr. XXXXXX XXXX. (Corresponding author: Ricardo Canute Kamikawachi.)

J. F. K., R. C. B. and R. C. K. are with the Universidade Tecnológica Federal do Paraná, 80230-901, Curitiba, Brazil; (e-mails: kuhne@alunos.utfpr.edu.br, baarreth@gmail.com and canute@utfpr.edu.br).

A. M. R. is with the Instituto de Telecomunicações, Campus Universitário de Santiago, 3810-193, Aveiro, Portugal; (e-mail: amrocha@av.it.pt). thinned, non-uniform thinned and micro-structured fibers [2][5]. Recently, Urrutia et al. published a comprehensive review of optical fiber refractometers were they compared the performance of interferometers, grating-based and resonance-based structures [6]. EFBG applications still draw the attention of many researchers [7]-[13]. Bekmurzayeva et al. proposed a biosensor based on an EFBG functionalized with aptamer as a ligand for thrombin detection [7]. Kumar et al. reported an EFBG coated with graphene oxide for ethanol detection in petrol showing a significant detection enhancement when compared with uncoated EFBG [8]. Corotti et al. reported an EFBG coated with diphenilalanine nanotubes capable to detect methanol in methanol-ethanol blend vapors [9]. Korganbayev et al. presented a compact sensor for multi-parametric measurements using a partially etched chirped fiber Bragg grating [10]. Bui et al. proposed a novel method for biochemical sensors using etched fiber Bragg gratings integrated in a fiber ring laser structure, without the need of a spectrometer [11]. For chemical and biochemical detection purposes, higher sensitivities are an important feature for obtaining detection limits in the order of $\mathrm{ng} / \mathrm{mL}$. Shivananju et al. studied EFBG coated with polyelectrolytes thin films, using computational simulation and experimental measurements [14]. They provided an estimation for the detection limit of the sensor considering the noise levels of the interrogation system. They found that sufficiently narrowed EFBGs can provide a competitive platform for real-time measurements of molecular interactions while 
simultaneously leveraging the high multiplexing capabilities of fiber optics. In previous works, our group showed that the refractive index sensitivity and the dynamic range of EFBG inscribed in multimode fibers are higher than those verified on single-mode fibers [15]. We have also shown that such gratings can be used in simultaneous measurements of refractive index and temperature [16]. These features make this device very promising for distributed sensing and/or multi-parameter measurements. Tsigaridas et al. presented a theoretical and experimental study on the effect of the etching process over the FBG properties [17]. They proposed a simple analytical expression between the effective refractive index and the radius of the etched FBG. Namiq and Ibsen presented a technique to measure the diameter of an optical fiber during the etching process using an FBG [18].

In this paper, we present a model developed from numerical simulations that allows estimating the surrounding refractive index response of an EFBG. Since the EFBG response curve depends on the fiber's characteristics (dimensions and material properties), the estimation of the response requires at least the determination of a reference curve. The results using the estimation models are compared with experimental measures, and show consistency within the experimental uncertainties. The results presented in this work are complementary to those reported by Tsigaridas et al. [17], and Namiq and Ibsen [18], and can be useful tools in EFBG analysis and design.

\section{Numerical Simulations and MOdELING}

In order to understand the EFBG response, a series of computational simulations were performed using the commercially finite element solver COMSOL Multiphysics ${ }^{\circledR}$ with the Wave Optics module.

Fig. 1 presents the effective refractive index $\left(n_{\text {eff }}\right)$ of the propagating modes as function of the fiber radius $r$, with surrounding refractive index (SRI) set to 1.31 RIU (in order to simulate an HF aqueous solution). Over the fiber radius interval from $6 \mu \mathrm{m}$ to $15 \mu \mathrm{m}$, the calculated $n_{\text {eff }}$ fits very well to a single exponential function, presented as Equation (1), as proposed by Tsigaridas et al. [17]. The fit results were $\eta=$ $(0.4246 \pm 0.0015) \mu \mathrm{m}^{-1}, n_{e_{0}}=(1.44616 \pm 0.00001) \mathrm{RIU}$, and $n_{e_{1}}=(-0.09792 \pm 0.00091)$ RIU. The maximum deviation of the simulated $n_{\mathrm{eff}}$ using this function was less than $3 \times 10^{-5}$ RIU. Adopting a grating period $\Lambda$ of $532.45 \mathrm{~nm}$, and the Bragg wavelength $\left(\lambda=2 n_{\text {eff }} \Lambda\right)$, it was possible to calculate the fiber radii corresponding to $1 \mathrm{~nm}$ wavelength shift intervals. These wavelength shifts values were chosen for the sake of comparison with the experimental results.

$$
n_{\mathrm{eff}}(r)=n_{e_{0}}+n_{e_{1}} \exp (-\eta r)
$$

Based on the results presented in Fig. 1, seven different radii were chosen to calculate $n_{\text {eff }}$ as a function of the SRI. Fig. 2 presents these results, with the SRI ranging from $1.310 \mathrm{RIU}$ to $1.445 \mathrm{RIU}$. For each radius, the calculated $n_{\text {eff }}$ fits very well to a non-linear function in the form of (2). In this equation, $n$ is the SRI, while $A, B, C$ and $k$ are parameters related to the fiber characteristics, such as core and cladding geometry and refractive index. The $k$ values were approximately the same

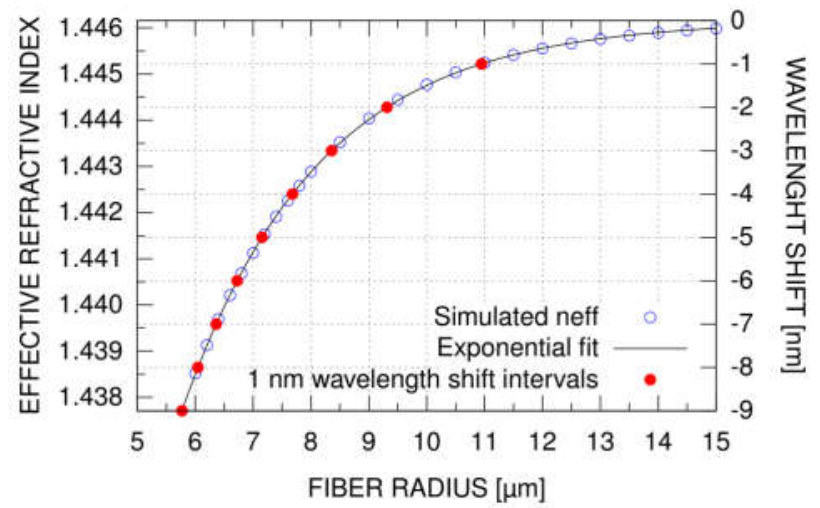

Fig. 1. Simulation of the effective refractive index as a function of the fiber radius. The simulation result was fit using (1). The corresponding Bragg wavelength is also presented in $1 \mathrm{~nm}$ intervals.

for all radii, with mean value of $0.3128 \pm 0.0026$. Through this, Equation (2) was used again to fit the simulated $n_{\text {eff }}$, but with $k$ fixed at 0.3128 . These fit results are presented in Fig. 2. The maximum deviation of the calculated $n_{\text {eff }}$ using (2) with this fixed $k$ was less than $1 \times 10^{-5}$ RIU. It is worth mentioning that our group has studied a simplified version of (2) with $k=1$ [16], which is still a good approximation. For the sake of comparison, the maximum deviation of the calculated $n_{\text {eff }}$ using that simpler equation $(k=1)$ was less than $3 \times 10^{-4}$ RIU.

$$
n_{\mathrm{eff}}(n)=A+\frac{B}{(C-n)^{k}}
$$

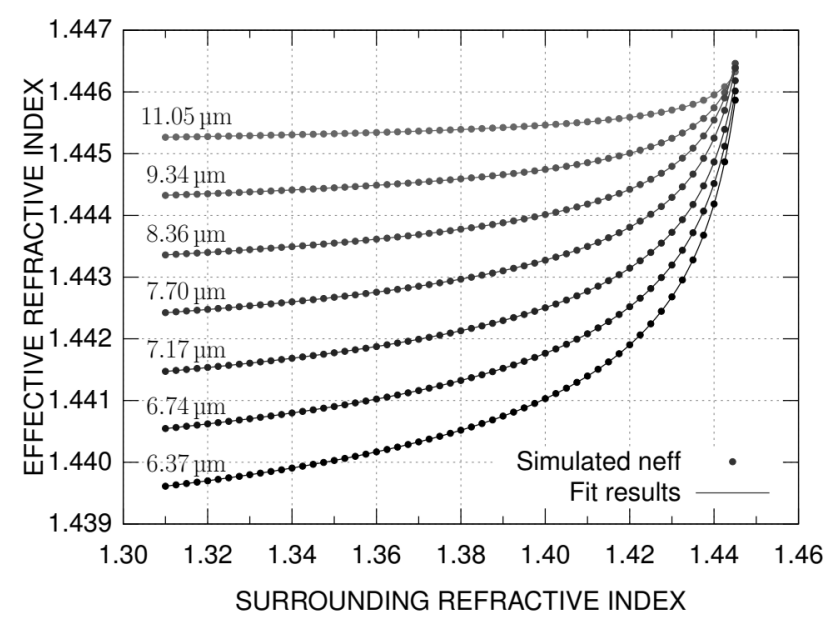

Fig. 2. Simulation of the effective refractive index as a function of the surrounding refractive index for seven different values of the fiber radius. The simulation results were fit using (2).

The dependence of $A, B$ and $C$ with the fiber radius is presented in Fig. 3. The $C$ parameter is linear with the fiber radius $r$, while both $A$ and $B$ present an exponential behaviour, which was expected after the results presented in Fig. 1.

The dependence on $r$ of $A, B$ and $C$ is presented in (3). By substituting $A(r), B(r)$ and $C(r)$ into (2), an equation with 9 parameters is derived, which can be used to describe $n_{\text {eff }}$ 

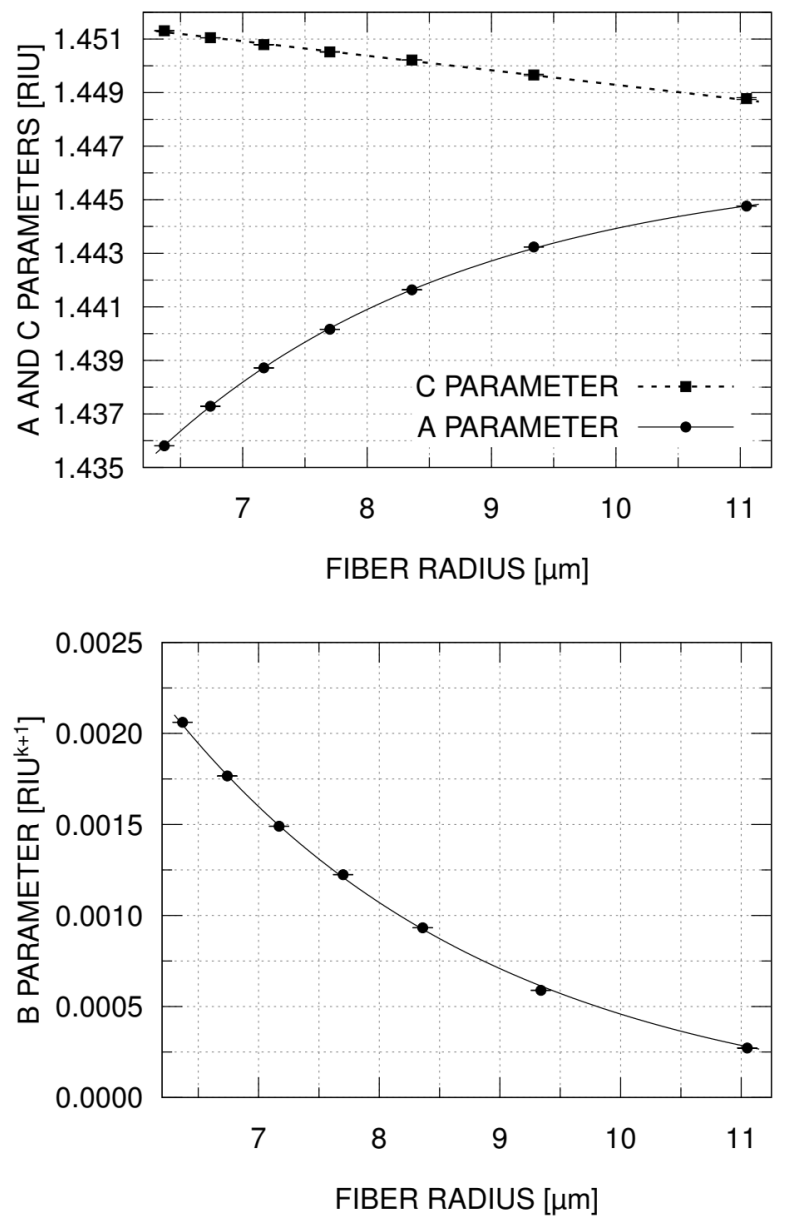

Fig. 3. Fit parameters from (2) over the data presented in Fig. 2 as a function of the fiber radius. These results were modelled using (3).

as a function of the SRI and the fiber radius, simultaneously. A surface fit is presented in Fig. 4, while Table I presents the corresponding fit parameters. The maximum deviation of the calculated $n_{\text {eff }}$ using the surface fit was less than $5 \times 10^{-5}$ RIU.

$$
\begin{aligned}
A(r) & =a_{0}+a_{1} \exp \left(-a_{2} r\right) \\
B(r) & =b_{0}+b_{1} \exp \left(-b_{2} r\right) \\
C(r) & =c_{0}+c_{1} r \\
n_{\mathrm{eff}}(n, r) & =A(r)+\frac{B(r)}{[C(r)-n]^{k}}
\end{aligned}
$$

Over the SRI range and fiber radii considered, the combined contribution of $b_{0}$ and $c_{1}$ is significantly small (about $2 \%$ to the $n_{\text {eff }}$ at $n=1.44$ ), and the exponents $a_{2}$ and $b_{2}$ differ for less than $10 \%$. This means that the surface equation may be simplified by making $\eta=a_{2}=b_{2}, b_{0}=0$ and $c_{1}=0$, and this is presented as (4). The maximum deviation of the calculated $n_{\text {eff }}$ using (4) was less than $2 \times 10^{-4}$ RIU, and is significantly lesser for smaller SRI values. This is consistent with the results presented in Fig. 1, since Equations (1) and (4) share the same functional structure for fixed SRI, whose equivalence is presented in (5). In this equation, $n_{c}$ refers to

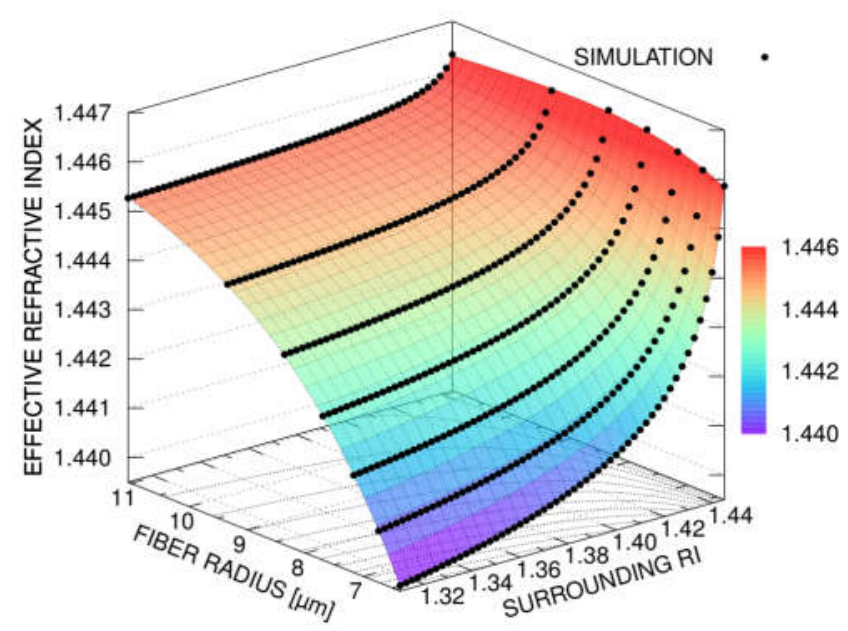

Fig. 4. Simulated $\boldsymbol{n}_{\text {eff }}$ surface as a function of the fiber radius and the surrounding refractive index. The surface fit was done using (3).

the SRI during the cladding etching $\left(n_{c}=1.31\right)$. In order to compare with the results of (3), the fit results using (4) are also presented in Table I.

$$
\begin{aligned}
n_{\mathrm{eff}}(n, r)= & a_{0}+\left[a_{1}+\frac{b_{1}}{\left(c_{0}-n\right)^{k}}\right] \exp (-\eta r) \\
& n_{e_{0}} \equiv a_{0} \\
& n_{e_{1}} \equiv a_{1}+\frac{b_{1}}{\left(c_{0}-n_{c}\right)^{k}}
\end{aligned}
$$

\section{A. Estimating the sensor's response}

Equation (4) is useful to estimate the change in the $n_{\text {eff }}$ for two different $r$ values at the same $n$, which is presented as (6).

$$
\begin{aligned}
\Delta_{r} n_{\mathrm{eff}} & =n_{\mathrm{eff}}(n, r)-n_{\mathrm{eff}}\left(n, r_{0}\right) \\
& =\left[a_{1}+\frac{b_{1}}{\left(c_{0}-n\right)^{k}}\right]\left[\exp (-\eta r)-\exp \left(-\eta r_{0}\right)\right]
\end{aligned}
$$

Therefore, for a pair of EFBG which differ only by their fiber radius, if (4) is a good approximation, then (6) may be used to estimate the relative change in $\Delta_{r} n_{\mathrm{eff}}$ as a function of $n$. For convenience, $n_{c}$ will be used as SRI reference, and this is presented at (7). In this equation, the denominator was substituted by $n_{e_{1}}$, from (5). The remarkable property of (7) is that it does not explicitly depend on $r$.

$$
\begin{aligned}
\frac{\Delta_{r} n_{\mathrm{eff}}(n)}{\Delta_{r} n_{\mathrm{eff}}\left(n_{c}\right)} & =\frac{a_{1}+\frac{b_{1}}{\left(c_{0}-n\right)^{k}}}{a_{1}+\frac{b_{1}}{\left(c_{0}-n_{c}\right)^{k}}} \\
& =\frac{1}{n_{e_{1}}}\left[a_{1}+\frac{b_{1}}{\left(c_{0}-n\right)^{k}}\right]
\end{aligned}
$$

Since the Bragg wavelength is proportional to $n_{\mathrm{eff}}, \Delta_{r} \lambda$ is also proportional to $\Delta_{r} n_{\mathrm{eff}}$. Through this, the wavelength 
TABLE I

Fit RESUlts of Equations (3) AND (4) OVER THE SIMULATED $\boldsymbol{n}_{\mathrm{eff}}$.

\begin{tabular}{|cc|cc|c|}
\hline \multicolumn{2}{|c|}{ Equation (3) } & \multicolumn{2}{c|}{ Equation (4) } & \\
\hline Parameter & Value & Parameter & Value & Unit \\
\hline$k$ & $0.3120 \pm 0.0087$ & $k$ & $0.3122 \pm 0.0141$ & - \\
$a_{0}$ & $1.44643 \pm 0.00002$ & $a_{0}$ & $1.44612 \pm 0.00001$ & $\mathrm{RIU}$ \\
$a_{1}$ & $-0.1347 \pm 0.0017$ & $a_{1}$ & $-0.1499 \pm 0.0026$ & $\mathrm{RIU}$ \\
$a_{2}$ & $0.3987 \pm 0.0018$ & $\eta$ & $0.4200 \pm 0.0009$ & $\mu \mathrm{m}^{-1}$ \\
$b_{0}$ & $-0.00014 \pm 0.00001$ & & - neglected - & $\mathrm{RIU}^{1}+\mathrm{k}$ \\
$b_{1}$ & $0.0221 \pm 0.0011$ & $b_{1}$ & $0.0261 \pm 0.0021$ & $\mathrm{RIU}^{1}+\mathrm{k}$ \\
$b_{2}$ & $0.3626 \pm 0.0034$ & & - same as $\eta-$ & $\mu \mathrm{m}^{-1}$ \\
$c_{0}$ & $1.45462 \pm 0.00038$ & $c_{0}$ & $1.45087 \pm 0.00030$ & $\mathrm{RIU}$ \\
$c_{1}$ & $-0.00053 \pm 0.00003$ & & - neglected & $\mathrm{RIU} / \mu \mathrm{m}$ \\
\hline
\end{tabular}

difference of a fiber pair can be mapped in terms of $\Delta_{r} \lambda\left(n_{c}\right)$ and $n$, which is presented in (8). In this, $\Delta_{r} \lambda\left(n_{c}\right)$ is simply represented as $\Delta \lambda_{c}$

$$
\Delta_{r} \lambda(n)=\frac{1}{n_{e_{1}}}\left[a_{1}+\frac{b_{1}}{\left(c_{0}-n\right)^{k}}\right] \Delta \lambda_{c}
$$

At this approximation, if we compare two fibers which differ only by their radii, at the same SRI, their wavelength difference from the same reference should be proportional. This result is presented in (9).

$$
\frac{\Delta_{r_{2}} \lambda(n)}{\Delta_{r_{1}} \lambda(n)}=\frac{\Delta \lambda_{c_{2}}}{\Delta \lambda_{c_{1}}} \Longrightarrow \Delta_{r_{2}} \lambda(n)=\frac{\Delta \lambda_{c_{2}}}{\Delta \lambda_{c_{1}}} \Delta_{r_{1}} \lambda(n)
$$

Equation (9) suggests that it is possible to estimate the wavelength response of a EFBG $\left(\lambda_{2}\right)$ by knowing the response of a similar one $\left(\lambda_{1}\right)$, and the ratio of their wavelength shift during the cladding removal (at $n=n_{c}$ ). An obvious reference to be used in (9) is $\lambda_{0}$ for no cladding removal, which is insensitive to the SRI, that is, $n_{\mathrm{eff}} \rightarrow n_{e_{0}} \Longrightarrow \lambda_{0}=2 n_{e_{0}} \Lambda$. By using this reference, Equation (9) can be rewritten as (10), and $\Delta \lambda_{c}$ must be reinterpreted as the wavelength shift during the cladding removal.

$$
\lambda_{2}(n)=\lambda_{0}\left(1-\frac{\Delta \lambda_{c_{2}}}{\Delta \lambda_{c_{1}}}\right)+\frac{\Delta \lambda_{c_{2}}}{\Delta \lambda_{c_{1}}} \lambda_{1}(n)
$$

Equation (10) can be used in interpolation. If one knows the response curve for $\lambda_{1}$, and $\lambda_{2}$ at two different SRI, then by solving $\lambda_{2}=h_{0}+h_{1} \lambda_{1}$ for $h_{0}$ and $h_{1}$, it is possible to estimate $\lambda_{2}$ over the same SRI range. Additionally, we can remove the first term in the right side of (10) by subtracting the wavelength at another SRI reference $\left(n_{0}\right)$, resulting in (11).

$$
\lambda_{2}(n)-\lambda_{2}\left(n_{0}\right)=\frac{\Delta \lambda_{c_{2}}}{\Delta \lambda_{c_{1}}}\left[\lambda_{1}(n)-\lambda_{1}\left(n_{0}\right)\right]
$$

Equation (11) can be used in extrapolation. By knowing the response curve for $\lambda_{1}$, the wavelength shift of $\lambda_{1}$ and $\lambda_{2}$ during the cladding removal, and $\lambda_{2}$ at $n_{0}$, then it is possible to estimate $\lambda_{2}$ over the same SRI range of $\lambda_{1}$.

\section{B. Interpolation procedure}

1) For a first sensor, construct its wavelength response curve (wavelength vs SRI) using the experimental data fitting with (12). This equation relates to (2) by $A^{\prime}=$ $2 A \Lambda$ and $B^{\prime}=2 B \Lambda$;

$$
\lambda_{1}(n)=A^{\prime}+\frac{B^{\prime}}{(C-n)^{k}}
$$

2) For the first, and a second sensor with same characteristics except the fiber radius after the cladding removal, measure their wavelength response at two different SRI values $n_{a}$ and $n_{b}$. Let these wavelengths be $\lambda_{1 a}$ and $\lambda_{1 b}$ for the first sensor, and $\lambda_{2 a}$ and $\lambda_{2 b}$ for the second one, respectively;

3) From (10), expressed as $\lambda_{2}=h_{0}+h_{1} \lambda_{1}$, solve the following linear system for $h_{0}$ and $h_{1}$ :

$$
\left[\begin{array}{l}
\lambda_{2 a}=h_{0}+h_{1} \lambda_{1 a} \\
\lambda_{2 b}=h_{0}+h_{1} \lambda_{1 b}
\end{array}\right] ;
$$

4) Finally, the second sensor's wavelength response curve is given by:

$$
\lambda_{2 i}(n)=h_{0}+h_{1} \lambda_{1}(n),
$$

where the $i$ index refers to the interpolation procedure.

\section{Extrapolation procedure}

1) For a first sensor, measure its wavelength shift during the cladding removal, $\Delta \lambda_{c_{1}}$;

2) Follow the same first step of the Interpolation procedure, that is, for the first sensor, construct its wavelength response curve $\lambda_{1}(n)$ using the experimental data fitting with (12);

3) For a second sensor, measure its wavelength shift during the cladding removal, $\Delta \lambda_{c_{2}}$;

4) For the first and second sensor, measure their wavelength responses at some SRI reference $n_{a}$. Let these wavelengths be $\lambda_{1 a}$ and $\lambda_{2 b}$, for the first and second sensor, respectively;

5) Finally, the second sensor's wavelength response curve is given by:

$$
\lambda_{2 e}(n)=\lambda_{2 a}+\frac{\Delta \lambda_{c_{2}}}{\Delta \lambda_{c_{1}}}\left[\lambda_{1}(n)-\lambda_{1 a}\right],
$$

where the $e$ index refers to the extrapolation procedure. 


\section{Testing the estimation models}

In order to test these estimation models, it was used the same radii of Fig. 2, whose values are presented in Table II. The SRI reference was $n_{0}=n_{c}=1.31$. The effective refractive index $n_{\text {eff }}$ was calculated using (3). The wavelength $\lambda_{c}$ was obtained from the calculated $n_{\text {eff }}$, considering $\Lambda=532.45 \mathrm{~nm}$. The reference for $\Delta \lambda_{c}$ was $\lambda_{0}=2 n_{e_{0}} \Lambda=(1540.014 \pm 0.008) \mathrm{nm}$, with $n_{e_{0}}=$ $(1.44616 \pm 0.00001)$ RIU, obtained from the fit using (1), which was presented in Fig. 1. For the interpolation, the second reference for $\lambda_{2}$ was arbitrarily chosen as $n=1.43$. Fig. 5 presents the wavelength shift estimation for the seven radii presented in Table II. The response curve used as reference for both extrapolation and interpolation was $r_{4}=7.70 \mu \mathrm{m}$ with $\Delta \lambda_{c_{4}}=-3.97 \mathrm{~nm}$ (fourth row). Table II also presents the estimation results. In general, both maximum deviation $\left(\delta_{\max }\right)$ and standard deviation $(\sigma)$ for the extrapolation are higher than the interpolation. The higher deviations occur with the lower radii, which present higher sensitivity.
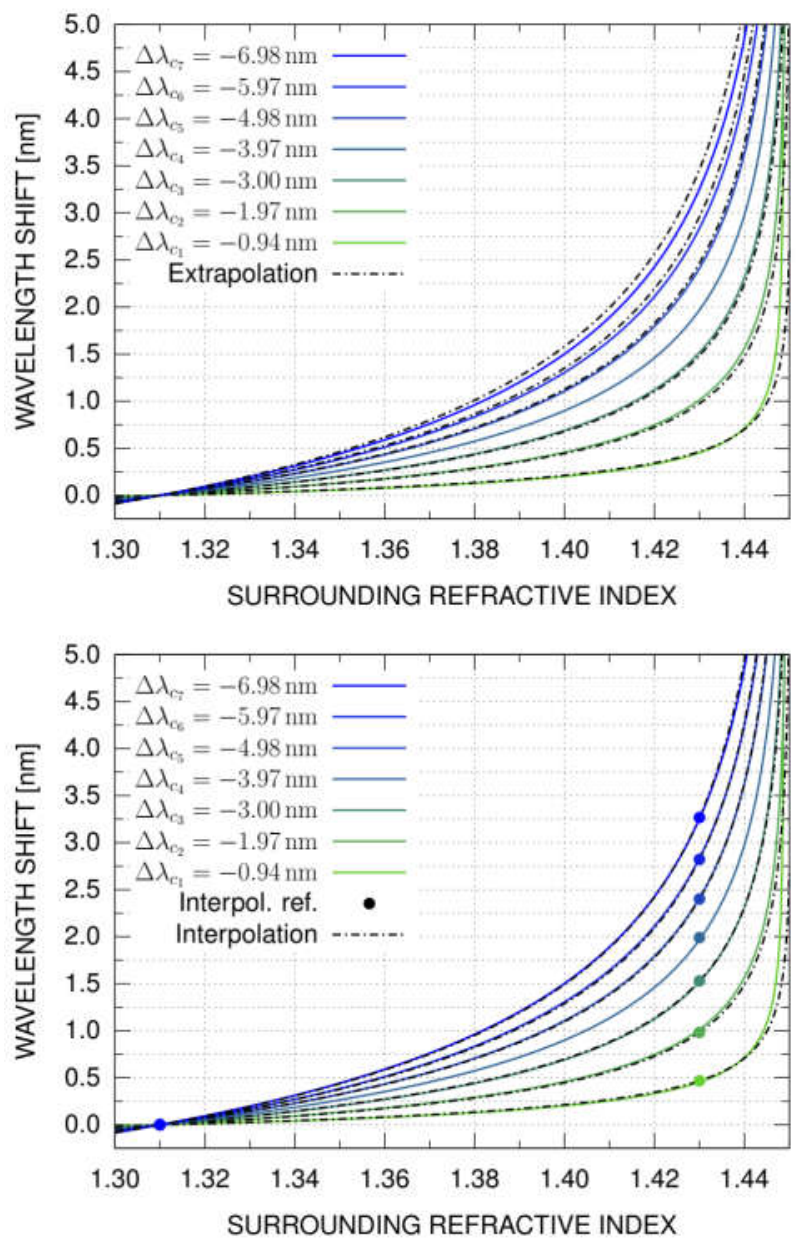

Fig. 5. Extrapolation (top) and interpolation (bottom) of the wavelength response simulated for seven different fiber radii. Solid line represents the simulation data fitting with (12). The wavelength response references used in the interpolation procedure (second step) are represented with circles.

\section{Materials and Methods}

In order to test the estimation models, 7 FBGs were inscribed and the cladding were reduced using different etching times to present different refractive index sensibilities. The fiber gratings were inscribed in a single mode fiber (Draka, G.652) using the phase-mask technique, with an ArF excimer laser (Coherent Xantos 500XS) emitting at $193 \mathrm{~nm}$. An Ibsen photonics phase mask with $1073.5 \mathrm{~nm}$ pitch was used. The exposure time was $4 \mathrm{~min}$, with pulse energy of $4 \mathrm{~mJ}$ at $250 \mathrm{~Hz}$ repetition rate. The chemical etching of the cladding was performed in aqueous solution of hydrofluoric acid (HF). The reaction was stopped by the fiber immersion in water, and neutralized by its immersion in sodium hydroxide $(\mathrm{NaOH})$ solution. The etching process was performed with 7 fibers, and stopped after observing wavelength shifts from $1 \mathrm{~nm}$ to $7 \mathrm{~nm}$, in $1 \mathrm{~nm}$ intervals. These 7 gratings were named as $F B G_{n}$, with $n$ ranging from 1 to 7 . After each etching, the fibers were immersed in water at room temperature $(n=$ 1.333) in order to register a wavelength reference and the corresponding wavelength shift due to the cladding removal $\left(\Delta \lambda_{c}\right)$. All measurements were realized using an Optical Spectrum Analyser-OSA (Yokogawa, AQ6375), with $\pm 5 \mathrm{pm}$ of wavelength stability, and an ASE as light source. The refractive index responses were determined by immersing the gratings into glycerine-water blends, with different concentrations. Glycerine-water blends were prepared by adding glycerine and water in different proportions, resulting in samples refractive index range from 1.3330 to 1.4580 . To avoid cross-sensitivity effects, the gratings were kept at $(22.0 \pm 0.5){ }^{\circ} \mathrm{C}$ with minimal mechanical deformations during the experiments. The refractive indexes of the samples were measured at the same temperature with an Abbe refractometer (Biobrix - 2WAJ) with $2 \times 10^{-4}$ resolution, operating in sodium D-line at $589 \mathrm{~nm}$. The measurements were performed 5 times under repeatability conditions.

\section{RESULtS AND Discussions}

Chemical etching is an exothermic process that initially causes a red shift in the wavelength $\left(\Delta \lambda_{R S}\right)$. However, as the evanescent field penetrates the external medium, the wavelength presents a blue shift. Fig. 6 presents this process for $F B G_{2}$. After the wavelength shift returned to zero, the acid solution is replaced by a lower concentration one in order to reduce the etching speed and to have a better control of the process. Through this, it was possible to construct seven different sensors with $\Delta \lambda_{c}$ ranging from $-1 \mathrm{~nm}$ to $-7 \mathrm{~nm}$, in approximately $1 \mathrm{~nm}$ intervals. Table III presents the initial wavelength responses $\lambda_{0}$, the wavelength shift due to the cladding removal $\Delta \lambda_{c}$ (using water as reference), and the observed red shift during the etching $\Delta \lambda_{R S}$ for each sensor. Theoretically, $\lambda_{0}$ and $\Delta \lambda_{R S}$ should be the same for all sensors, since the fiber model, the grating period and experimental procedure were the same. Also, $\Delta \lambda_{R S}$ should be proportional to the temperature change, which depends on the reaction rate, and consequently the acid concentration. For all sensors, the "high concentration" stage of the chemical etching was performed in aqueous solution of $\mathrm{HF}$ at $40 \%$. However, 
TABLE II

PARAMETERS AND RESULTS FOR THE ESTIMATION MODELS APPLIED OVER SEVEN DIFFERENT FIBER RADII. EXTRAPOLATION USES THE WAVELENGTH SHIFT DURING THE CLADDING REMOVAL $\left(\boldsymbol{\Delta} \boldsymbol{\lambda}_{\boldsymbol{c}}\right)$. THE VALUES FOR $\boldsymbol{\delta}_{\max }$ AND $\boldsymbol{\sigma}$ WERE CALCULATED WITH THE SRI RANGING FROM 1.310 RIU TO 1.445 RIU.

\begin{tabular}{|c|c|c|c|c|c|c|c|c|}
\hline \multirow[b]{2}{*}{$i$} & \multirow[b]{2}{*}{$r[\mu \mathrm{m}]$} & \multirow[b]{2}{*}{$n_{\text {eff }}[\mathrm{RIU}]$} & \multirow[b]{2}{*}{$\lambda_{c}[\mathrm{~nm}]$} & \multirow[b]{2}{*}{$\Delta \lambda_{c}[\mathrm{~nm}]$} & \multicolumn{2}{|c|}{ Extrapolation } & \multicolumn{2}{|c|}{ Interpolation } \\
\hline & & & & & $\delta_{\max }[\mathrm{nm}]$ & $\sigma[\mathrm{nm}]$ & $\delta_{\max }[\mathrm{nm}]$ & $\sigma[\mathrm{nm}]$ \\
\hline 1 & 11.05 & 1.44528 & 1539.08 & -0.94 & 0.10 & 0.01 & 0.09 & 0.01 \\
\hline 2 & 9.34 & 1.44431 & 1538.05 & -1.97 & 0.20 & 0.03 & 0.18 & 0.03 \\
\hline 3 & 8.36 & 1.44334 & 1537.02 & -3.00 & 0.14 & 0.02 & 0.04 & 0.01 \\
\hline 4 & 7.70 & 1.44243 & 1536.04 & -3.97 & \multicolumn{2}{|c|}{ - reference - } & \multicolumn{2}{|c|}{ - reference - } \\
\hline 5 & 7.17 & 1.44149 & 1535.04 & -4.98 & 0.19 & 0.03 & 0.06 & 0.01 \\
\hline 6 & 6.74 & 1.44055 & 1534.04 & -5.97 & 0.42 & 0.07 & 0.14 & 0.02 \\
\hline 7 & 6.37 & 1.43960 & 1533.03 & -6.98 & 0.69 & 0.12 & 0.29 & 0.04 \\
\hline
\end{tabular}

given the characteristic experimental uncertainties, these wavelengths fluctuate with mean values $\overline{\lambda_{0}}=(1552.12 \pm 0.15) \mathrm{nm}$ and $\overline{\Delta \lambda_{R S}}=(0.72 \pm 0.02) \mathrm{nm}$. The uncertainties in the wavelength shifts are expected to be lesser than those of the absolute wavelength $\left(\lambda_{0}\right)$ due to cancellation of systematic error. Therefore, we can assume the uncertainty for each $\Delta \lambda_{c}$ value was at least $0.02 \mathrm{~nm}$. In addition, it is worth mentioning that the reduction of the fiber diameter changes the guidance conditions allowing the existence of higher order modes, as observed by Ayupova et al. [19]. However, these modes had a very small intensity and were not analyzed in this work.

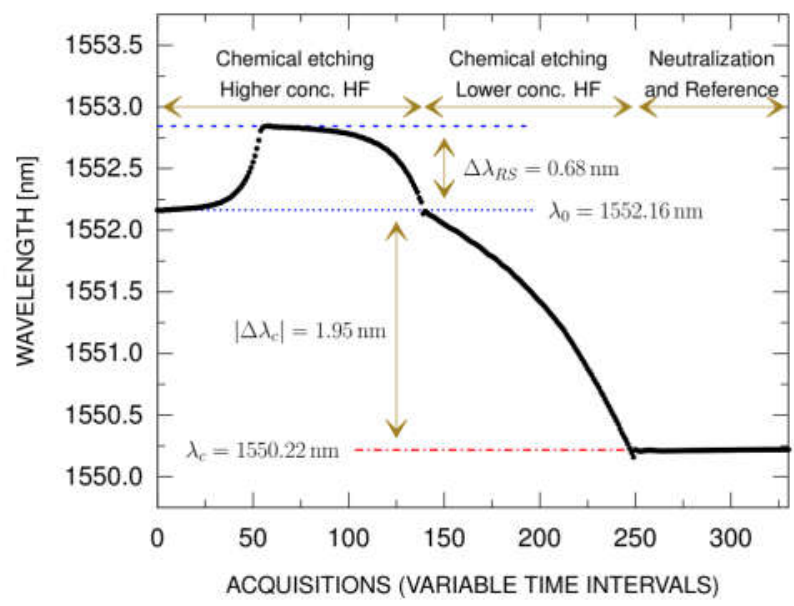

Fig. 6. Wavelength response during the etching process for $\boldsymbol{F} B \boldsymbol{G}_{2}$. After a red shift $\left(\Delta \lambda_{R S}\right)$, the reduction of the cladding results in a blue shift. The etching was performed until the $\Delta \boldsymbol{\lambda}_{\boldsymbol{c}}$ was approximately equal to $-2 \mathrm{~nm}$.

The wavelength as a function of the refractive index of the 7 EFBGs are shown in Fig. 7. In this, the response curves were fit using (12), and the fit parameters are presented in Table IV. These curves were determined in 2 steps. First, for each EFBG, all four parameters of (12) were optimized. The mean characteristic exponent was determined as $k=$ $0.101 \pm 0.008$, and it was fixed for all EFBGs in the next step, when the parameters $A^{\prime}, B^{\prime}$ and $C$ were optimized again. The asymmetric wavelength shifts between the curves presented in Fig. 7 are consistent with the experimental $\Delta \lambda_{c}$ values presented in Table III and Fig. 8, and they contrast with the symmetry presented by the simulation results (Fig. 2).
TABLE III

REFERENCE WAVELENGTHS FOR THE SEVEN FBG SENSORS ETCHED WITH PROGRESSIVE $\boldsymbol{\Delta} \boldsymbol{\lambda}_{\boldsymbol{c}}$. THE UNCERTAINTIES ARE $0.15 \mathrm{~nm}$ FOR $\boldsymbol{\lambda}_{\mathbf{0}}$, AND $0.02 \mathrm{~nm}$ FOR $\boldsymbol{\Delta} \boldsymbol{\lambda}_{\boldsymbol{c}}$ AND $\boldsymbol{\Delta} \boldsymbol{\lambda}_{\boldsymbol{R}} \boldsymbol{S}$.

\begin{tabular}{|lccc|}
\hline Sensor & $\lambda_{0}[\mathrm{~nm}]$ & $\Delta \lambda_{c}[\mathrm{~nm}]$ & $\Delta \lambda_{R S}[\mathrm{~nm}]$ \\
\hline$F B G_{1}$ & 1551.92 & -1.00 & 0.73 \\
$F B G_{2}$ & 1552.16 & -1.95 & 0.68 \\
$F B G_{3}$ & 1552.04 & -2.95 & 0.72 \\
$F B G_{4}$ & 1552.00 & -3.93 & 0.73 \\
$F B G_{5}$ & 1552.15 & -5.07 & 0.73 \\
$F B G_{6}$ & 1552.37 & -5.95 & 0.72 \\
$F B G_{7}$ & 1552.16 & -7.12 & 0.73 \\
\hline
\end{tabular}

The $C$ parameter was approximately constant with $C=$ $(1.4612 \pm 0.0007) \mathrm{RIU}$. The results for $A^{\prime}$ and $B^{\prime}$ are presented in Fig. 8 as a function of $\Delta \lambda_{c}$. As (10) suggests, $A^{\prime}$ and $B^{\prime}$ have a strict linear dependence with the wavelength shift due to the cladding removal $\Delta \lambda_{c}$. As $\Delta \lambda_{c} \rightarrow 0$ it was expected to $B^{\prime} \rightarrow 0$, however $B^{\prime}=0$ at $\Delta \lambda_{c}=(0.73 \pm 0.03) \mathrm{nm}$ through extrapolation, which coincides with the observed red shift $\Delta \lambda_{R S}$ during the etching. This suggests the etching process does not simply reduces the cladding, but also result in an annealing effect changing the wavelength permanently. Through this, the measured $\Delta \lambda_{c}$ should be corrected, and our best estimation for that is subtracting the red shifts $\Delta \lambda_{R S}$ observed during the etching.

TABLE IV

FIT RESULTS FOR EACH EFBG SENSOR USING (12) OVER THE DATA PRESENTED IN FIG. 7.

\begin{tabular}{|cccc|}
\hline Sensor & $A^{\prime}[\mathrm{nm}]$ & $B^{\prime}\left[\mathrm{nm} \cdot \mathrm{RIU}^{\mathrm{k}}\right]$ & $C[\mathrm{RIU}]$ \\
\hline$F B G_{1}$ & $1547.67 \pm 0.08$ & $2.60 \pm 0.06$ & $1.4613 \pm 0.0006$ \\
$F B G_{2}$ & $1545.02 \pm 0.26$ & $4.16 \pm 0.21$ & $1.4618 \pm 0.0014$ \\
$F B G_{3}$ & $1542.30 \pm 0.10$ & $5.51 \pm 0.08$ & $1.4609 \pm 0.0003$ \\
$F B G_{4}$ & $1539.48 \pm 0.09$ & $6.90 \pm 0.07$ & $1.4607 \pm 0.0002$ \\
$F B G_{5}$ & $1536.36 \pm 0.23$ & $8.67 \pm 0.18$ & $1.4611 \pm 0.0007$ \\
$F B G_{6}$ & $1534.02 \pm 0.24$ & $10.00 \pm 0.19$ & $1.4616 \pm 0.0005$ \\
$F B G_{7}$ & $1530.29 \pm 0.15$ & $11.83 \pm 0.13$ & $1.4609 \pm 0.0006$ \\
\hline
\end{tabular}

\section{A. Estimating the experimental results}

Fig. 9 presents the estimation of the experimental wavelength response of the EFBGs, through interpolation and extrapolation, using Equations (14) and (15), respectively. 


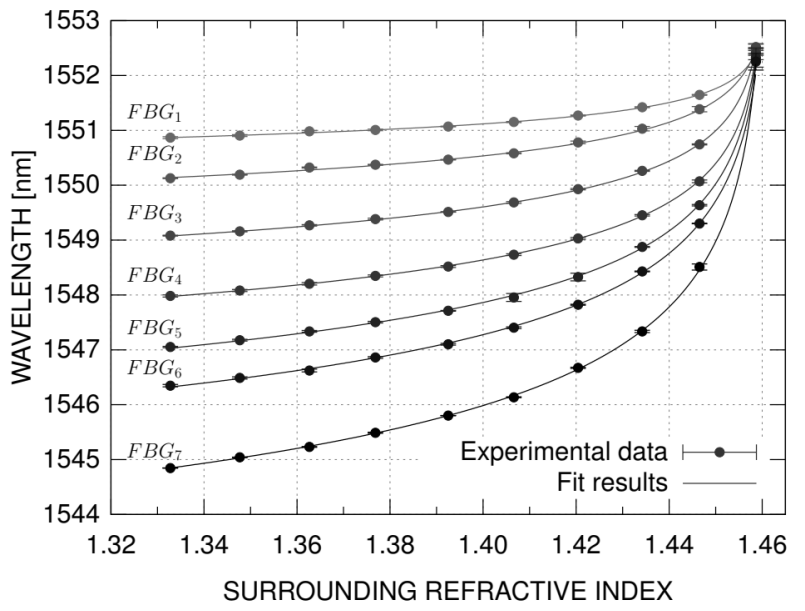

Fig. 7. Wavelength response for the 7 EFBGs as a function of the refractive index.
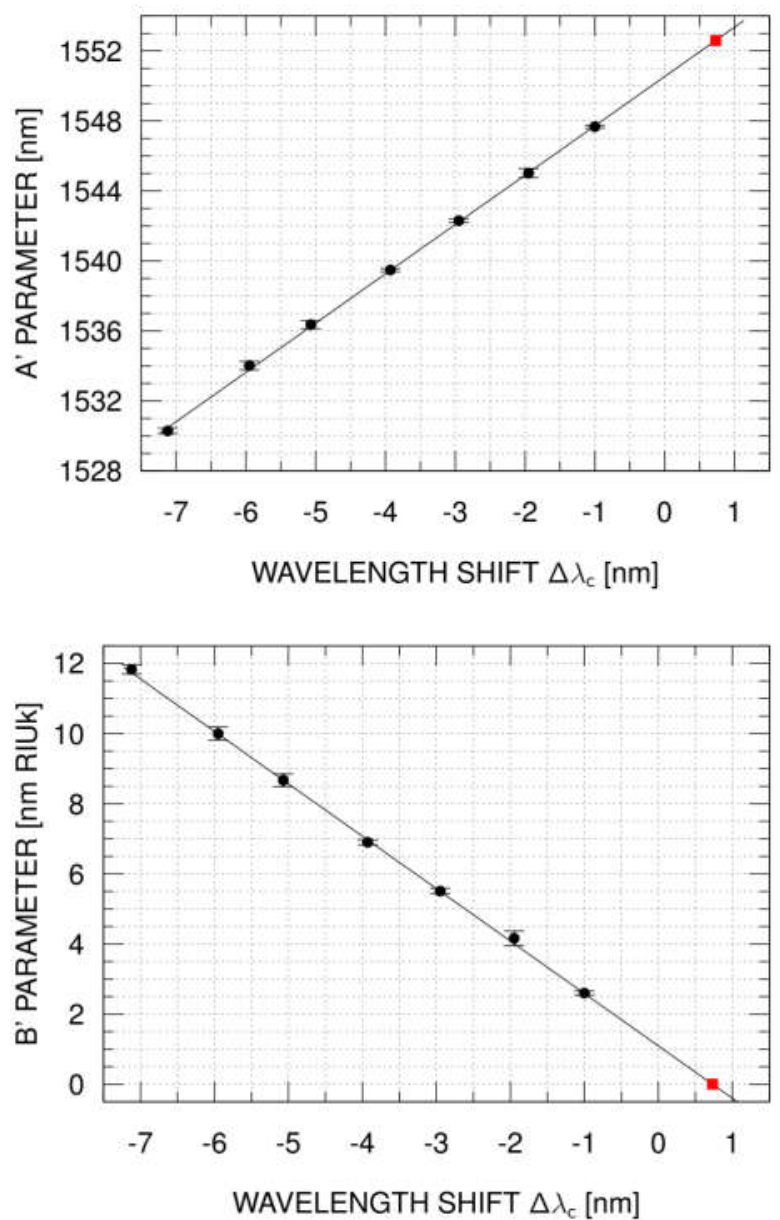

Fig. 8. Fit parameters $\left(\boldsymbol{A}^{\prime}\right.$ and $\left.\boldsymbol{B}^{\prime}\right)$ from (12) over the data presented in Fig. 7 as a function of the wavelength shift due to the cladding removal $\left(\Delta \lambda_{c}\right)$. The red squares represent the extrapolation for $\boldsymbol{B}^{\prime} \rightarrow 0$, when the sensor should be insensitive to the SRI. This value correspond to $\boldsymbol{\Delta} \boldsymbol{\lambda}_{\boldsymbol{c}}=(0.73 \pm 0.03) \mathrm{nm}$ and $\boldsymbol{A}^{\prime}=(1552.59 \pm 0.08) \mathrm{nm}$

The wavelength shift of $F B G_{4}$ was used as the calibration
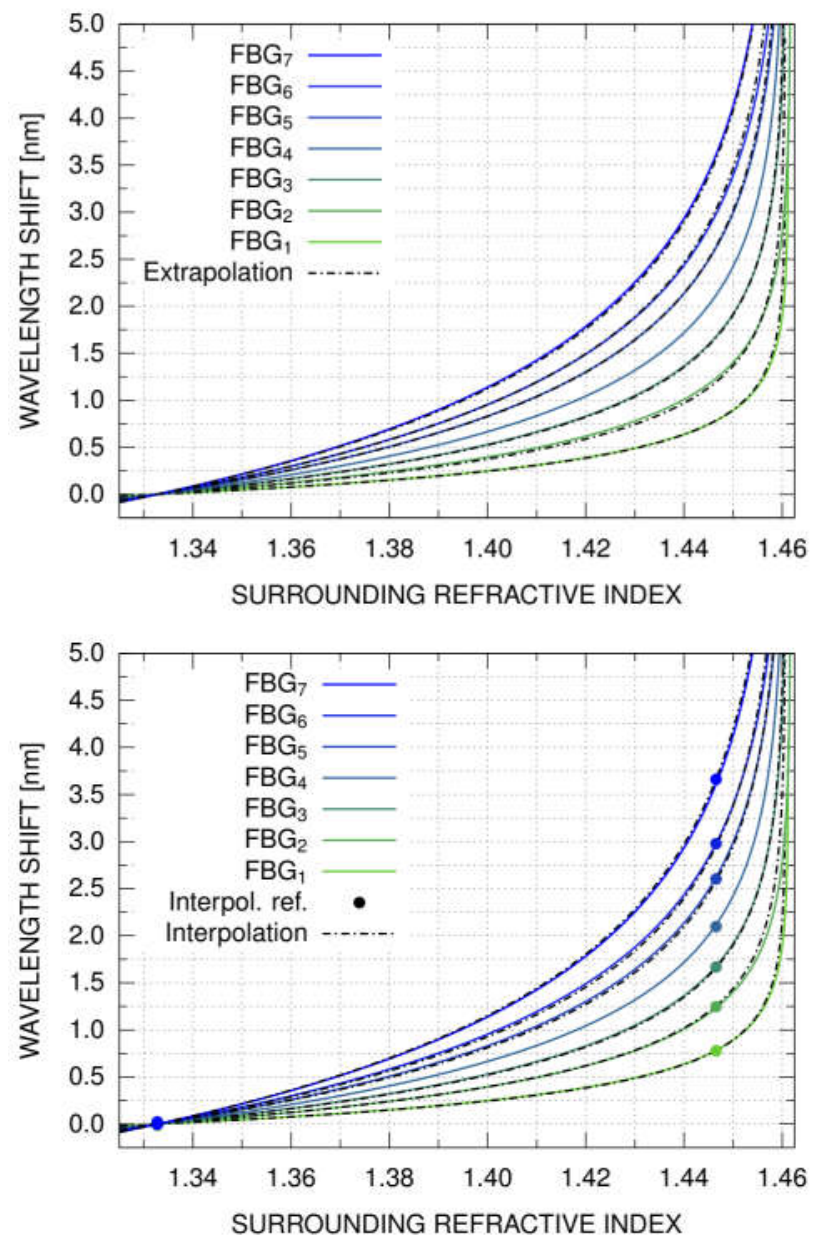

Fig. 9. Extrapolation (top) and interpolation (bottom) of the wavelength response for seven EFBGs. Solid line represents the experimental data fitting with (12). The wavelength response references used in the interpolation procedure (second step) are represented with circles.

curve. Water was the SRI reference for all wavelength shifts $\left(n_{a}=1.333 \mathrm{RIU}\right)$, since it was used to clean the fibers after the etching, determining $\Delta \lambda_{c}$ (Fig. 6). Table $\mathrm{V}$ presents the effective etching wavelength shift $\left(\Delta \lambda_{c}^{\prime}\right)$, which was determined as the wavelength shift due to the cladding removal subtracted by the red shift during the etching, $\Delta \lambda_{c}^{\prime}=\Delta \lambda_{c}-\Delta \lambda_{R S}$. This effective shift $\Delta \lambda_{c}^{\prime}$ was used in place of $\Delta \lambda_{c}$ in (15). The extrapolation procedure is simpler than the interpolation because it requires only the etching wavelength shifts $\Delta \lambda_{c}^{\prime}$ to estimate the wavelength response. The interpolation procedure is expected to have a better accuracy, but it requires at least another SRI reference $\left(n_{b}\right)$, which demands further measurements. For the interpolation procedure, the second SRI reference was chosen to be $n_{b}=1.447 \mathrm{RIU}$. Using these two SRI references, it was possible to calculate the $h_{1}$ parameter from (14). As expected, $h_{1} \approx \Delta \lambda_{c}^{\prime} / \Delta \lambda_{c_{r e f}}^{\prime}$, with maximum relative difference of $5 \%$ (for $F B G_{2}$ ).

The agreement between the estimated curves and the experimental data fitting was evaluated through maximum deviation $\left(\delta_{\max }\right)$ and standard deviation $(\sigma)$, considering the SRI interval from 1.333 RIU to $1.458 \mathrm{RIU}$. Table $\mathrm{V}$ presents $\delta_{\max }$ and 
TABLE V

RESULTS FOR THE ESTIMATION MODELS APPLIED OVER THE EFBG SENSORS. THE WAVELENGTH SHIFT DUE TO THE CLADDING REMOVAL WAS

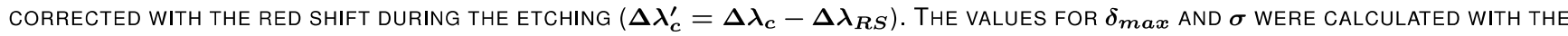
SRI RANGING FROM 1.333 RIU TO 1.458 RIU.

\begin{tabular}{|c|c|c|c|c|c|c|c|}
\hline & & \multicolumn{3}{|c|}{ Extrapolation } & \multicolumn{3}{|c|}{ Interpolation } \\
\hline Sensor & $\Delta \lambda_{c}^{\prime}[\mathrm{nm}]$ & $\delta_{\max }[\mathrm{nm}]$ & $\sigma[\mathrm{nm}]$ & $\Delta \lambda_{c_{i}}^{\prime} / \Delta \lambda_{c_{4}}^{\prime}$ & $\delta_{\max }[\mathrm{nm}]$ & $\sigma[\mathrm{nm}]$ & $h_{1}$ \\
\hline$F B G_{1}$ & -1.72 & 0.06 & 0.01 & 0.37 & 0.05 & 0.01 & 0.37 \\
\hline$F B G_{2}$ & -2.63 & 0.07 & 0.02 & 0.56 & 0.19 & 0.03 & 0.59 \\
\hline$F B G_{3}$ & -3.67 & 0.03 & 0.00 & 0.79 & 0.02 & 0.01 & 0.78 \\
\hline$F B G_{4}$ & -4.65 & \multicolumn{3}{|c|}{ - reference - } & \multicolumn{3}{|c|}{ - reference - } \\
\hline$F B G_{5}$ & -5.79 & 0.17 & 0.02 & 1.24 & 0.07 & 0.02 & 1.22 \\
\hline$F B G_{6}$ & -6.66 & 0.41 & 0.05 & 1.43 & 0.26 & 0.03 & 1.39 \\
\hline$F B G_{7}$ & -7.84 & 0.04 & 0.01 & 1.69 & 0.17 & 0.03 & 1.73 \\
\hline
\end{tabular}

$\sigma$ between the estimated and experimental curves responses for each $F B G$. In general, the results for extrapolation and interpolation are similar. The largest deviation occurred for $F B G_{6}$, which can be visualized in Fig. 9. However, when compared to the simulation (Fig. 5 and Table II), the accuracy of the estimation for the experimental results is embarrassingly good for both extrapolation and interpolation. These results show that the proposed estimation methods are useful and reliable to estimate the response curve of an EFBG using only a calibration curve of a similar sensor and few additional measurements.

\section{CONCLUSION}

In this work, an empirical mathematical model was proposed to describe the response curve of the EFBG external refractive index. The model was developed from the results of numerical simulations and it was verified experimentally. From this model, it was possible to construct two estimation approaches, in which the response curve of an EFBG could be predicted based on a reference calibration curve of a similar EFBG with a different etching. The extrapolation approach is simpler than the interpolation, since it only needs one value of surrounding refractive index for the wavelength response reference. However, the interpolation model provided better accuracy when compared to the experimental measurements. The proposed mathematical model and the estimation approaches can successfully predict EFBGs' response curves, which may be useful for the design of sensors with arbitrary sensitivity.

\section{ACKNOWLEDGMENT}

The used FBGs were fabricated at the Multi-User Photonics Facility-UTFPR-CT, associated laboratory of INCT FOTONICOM.

\section{REFERENCES}

[1] L. Dong, J. L. Cruz, L. Reekie, and L. Archambault, "Tuning and chirping fiber bragg gratings by deep etching," IEEE Photonics Technology Letters, vol. 7, no. 12, pp. 1433-1435, Dec 1995.

[2] A. Iadicicco, S. Campopiano, A. Cutolo, M. Giordano, and A. Cusano, "Microstructured fibre bragg gratings: analysis and fabrication," Electronics Letters, vol. 41, no. 8, pp. 466-468, April 2005.

[3] A. Iadicicco, A. Cusano, S. Campopiano, A. Cutolo, and M. Giordano, "Thinned fiber bragg gratings as refractive index sensors," IEEE Sensors Journal, vol. 5, no. 6, pp. 1288-1295, Dec 2005.
[4] A. Iadicicco, S. Campopiano, A. Cutolo, M. Giordano, and A. Cusano, "Nonuniform thinned fiber bragg gratings for simultaneous refractive index and temperature measurements," IEEE Photonics Technology Letters, vol. 17, no. 7, pp. 1495-1497, July 2005.

[5] A. Iadicicco, S. Campopiano, A. Cutolo, M. Giordano, and A. Cusano, "Refractive index sensor based on microstructured fiber bragg grating," IEEE Photonics Technology Letters, vol. 17, no. 6, pp. 1250-1252, June 2005

[6] A. Urrutia, I. Del Villar, P. Zubiate, and C. R. Zamarreño, "A comprehensive review of optical fiber refractometers: Toward a standard comparative criterion," Laser \& Photonics Reviews, vol. 13, no. 11, p. 1900094, 2019.

[7] A. Bekmurzayeva, K. Dukenbayev, M. Shaimerdenova, I. Bekniyazov, T. Ayupova, M. Sypabekova, C. Molardi, and D. Tosi, "Etched fiber bragg grating biosensor functionalized with aptamers for detection of thrombin," Sensors, vol. 18, no. 12, 2018. [Online]. Available: https://www.mdpi.com/1424-8220/18/12/4298

[8] P. Kumar, S. Kumar, J. Kumar, G. S. Purbia, O. Prakash, and S. K. Dixit "Graphene-oxide-coated fiber bragg grating sensor for ethanol detection in petrol," Measurement Science and Technology, vol. 31, no. 2, p. 025109 , nov 2019.

[9] R. D. P. Corotti, B. B. Cunha, R. C. Barreto, A. L. C. Conceição, and R. C. Kamikawachi, "Diphenylalanine nanotube coated fiber bragg grating for methanol vapor detection," IEEE Sensors Journal, vol. 20, no. 3, pp. 1290-1296, 2020.

[10] S. Korganbayev, T. Ayupova, M. Sypabekova, A. Bekmurzayeva, M. Shaimerdenova, K. Dukenbayev, C. Molardi, and D. Tosi, "Partially etched chirped fiber bragg grating (pecfbg) for joint temperature, thermal profile, and refractive index detection," Opt. Express, vol. 26, no. 14, pp. 18708-18720, Jul 2018. [Online]. Available: http://www.opticsexpress.org/abstract.cfm?URI=oe-26-14-18708

[11] H. Bui, T. B. Pham, V. A. Nguyen, V. D. Pham, T. C. Do, T. V. Nguyen, T. H. C. Hoang, H. T. Le, and V. H. Pham, "Novel method of dual fiber bragg gratings integrated in fiber ring laser for biochemical sensors," Measurement Science and Technology, vol. 29, no. 5, p. 055105, mar 2018.

[12] S. M. Pabbisetti Vayu Nandana Kishore, Sai Shankar Madhuvarasu, "Stimulus responsive hydrogel-coated etched fiber bragg grating for carcinogenic chromium (vi) sensing," Optical Engineering, vol. 57, no. 1 , pp. $1-7-7,2018$.

[13] W. Bao, X. Qiao, Q. Rong, and F. Chen, "Fiber-optic vector magnetometer based on magnetic fluid and fiber bragg grating written on a multi-clad fiber," IEEE Sensors Journal, vol. 18, no. 18, pp. 7486-7491, Sep. 2018.

[14] B. N. Shivananju, M. Renilkumar, G. R. Prashanth, S. Asokan, and M. M. Varma, "Detection limit of etched fiber bragg grating sensors," Journal of Lightwave Technology, vol. 31, no. 14, pp. 2441-2447, July 2013.

[15] J. F. Kuhne, A. M. Rocha, V. de Oliveira, H. J. Kalinowski, and R. C. Kamikawachi, "Experimental and numerical study on refractive index sensors based on fibre bragg gratings inscribed in multimode fibre," Measurement Science and Technology, vol. 29, no. 2, p. 025102, jan 2018.

[16] D. Fernandes, R. C. Barreto, A. G. Macedo, J. C. C. D. Silva, and R. C. Kamikawachi, "A simple equation to describe cross-sensitivity between temperature and refractive index in fiber bragg gratings refractometers," IEEE Sensors Journal, vol. 18, no. 3, pp. 1104-1110, Feb 2018.

[17] G. Tsigaridas, D. Polyzos, A. Ioannou, M. Fakis, and P. Persephonis 
"Theoretical and experimental study of refractive index sensors based on etched fiber bragg gratings," Sensors and Actuators A: Physical, vol. 209 , pp. $9-15,2014$.

[18] M. F. Namiq and M. Ibsen, "Simple technique of determining the fibre diameter during etching," Opt. Express, vol. 26, no. 25, pp. 3290832917 , Dec 2018

[19] T. Ayupova, M. Sypabekova, C. Molardi, A. Bekmurzayeva, M. Shaimerdenova, K. Dukenbayev, and D. Tosi, "Wavelet-based demodulation of multimode etched fiber bragg grating refractive index sensor," Sensors, vol. 19, no. 1, p. 39, Dec 2018.

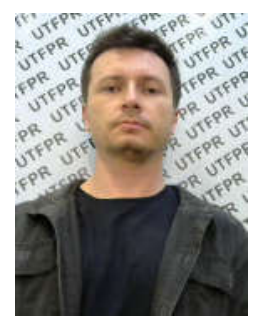

Jean Filipe Kuhne graduated in Industrial Electrical Engineering (2013), emphasis in Electronics / Telecommunications, with specialization in Industrial Automation (2015) from the Federal University of Technology - Paraná. Master (2015) and doctorate (in progress) in Sciences by the graduate program in Electrical and Computer Engineering at the Federal University of Technology - Paraná. Conducts research with optical sensors based on fiber Bragg gratings.

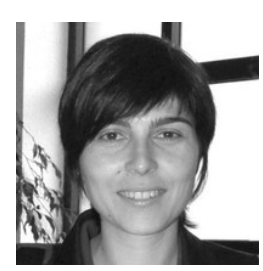

Ana Maria Rocha graduated in Physics Engineering from University of Aveiro in 2002 and completed her master degree in 2007 and $\mathrm{PhD}$ in 2012 both in Physics Engineering from University of Aveiro. At present, she is a Research Scientist in in Instituto de Telecomunicações, where she has been working in the Optical Components and Subsystems group. She has authored or co-authored $60+$ papers in international scientific journals and conference proceedings. She also collaborates with the Universidade de Aveiro as a lecture. Her main current research interests include high-capacity communications, Spatial Division Multiplexing system, wideband optical amplifiers, optical fiber devices and optical sensors. She is a member of the IEEE Photonics Society, the Optical Society of America and the the Portuguese Society of Optics and Photonics(SPOF)

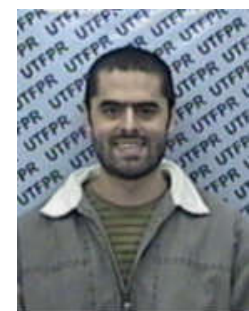

Rafael Carvalho Barreto received the B.Sc. degree in Physics from State University of Londrina (UEL) and the M.Sc. and Ph.D. degrees from the University of São Paulo (USP) in the field of atomic and molecular physics. He is currently a Professor at the Physics Department at UTFPR and he has experience on computational simulation, electronic structure calculation, modeling, simulation of liquids, computational physics and software development.

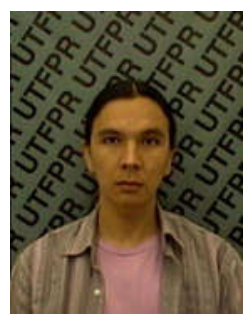

Ricardo Canute Kamikawachi received the B.Sc. in Physics from Federal University of Paraná (UFPR) and the M.Sc. and Ph.D. degrees (with sandwich period at the University of Aveiro in Portugal) from Federal University of Technology - Paraná (UTFPR). He is a member of the Brazilian Society of Microwave and Optoelectronics (SBMO) and the Brazilian Photonics Society (SBFoton). He is currently a Professor of the Physics Department at UTFPR, where he is developing fiber Bragg gratings sensors for physical, chemical and biochemical applications. 